\title{
Cognitive Training Game Versus Action Videogame: Effects on Cognitive Functions in Older Adults
}

\author{
Alexandra Perrot, $\mathrm{PhD}^{1,2}$ Pauline Maillot, $\mathrm{PhD}^{3}$, and Alan Hartley, $\mathrm{PhD}^{4}$
}

\begin{abstract}
Objective: The purpose of the study was to compare a cognitive training game, Kawashima Brain Training $(K B T)$, and an action videogame, Super Mario Bros $(S M B)$, in their effects on cognitive function in older adults.

Materials and Methods: Thirty-six older adults were randomly assigned to the KBT group, the SMB group, or the no-training no-contact control group. All participants completed several cognitive tests [matrix reasoning, Stroop, Trail Making Test, digit symbol substitution test (DSST), Corsi clock, spatial relation, and number comparison]. Then, participants in the game groups were instructed to play the videogame (KBT or SMB) for 1 hour, thrice per week, during 2 months, for a total training time of 24 hours. When the twenty-four 1-hour game sessions were complete, the three groups again completed the cognitive tests.

Results: Analysis of variances on each of the cognitive measures and Tukey's post hoc tests showed that the matrix reasoning change score was significantly greater in both game groups than in the control group. The Stroop test change was significantly greater in the KBT group than in control and SMB groups. The DSST, Corsi block test, spatial relations test, and number comparison test showed significantly greater change in the SMB group than in the control group with KBT intermediate.

Conclusion: The scope of benefits of SMB training seems broader than those from the KBT program. The intrinsic characteristics of SMB and KBT games may well be partly responsible for these differences.
\end{abstract}

Keywords: Cognitive training game, Action videogame, Cognitive functions, Aging

\section{Introduction}

$\mathbf{I}$ NTEREST IN INTERVENTIONS that can preserve or improve cognition in older adults has grown notably in the last decade. There is intuitive appeal to the notion that the challenges of new technologies such as videogames may have desirable incidental effects. Indeed, studies have shown that playing a videogame can lead to improved cognitive functions in the healthy elderly. ${ }^{1,2}$ After several weeks of videogame training, players showed improvements compared with control groups in processing speed, ${ }^{3}$ manual dexterity, ${ }^{4}$ visuomotor coordination, ${ }^{5}$ and executive functions. ${ }^{6}$

In attempts to capitalize on such results, several commercial game-like programs have been developed promising to strengthen and rejuvenate the brain [e.g., Kawashima Brain Training (KBT), Brain Age, Big Brain Academy, and Lumosity]. Despite claims about effects on the brain we will use the more neutral term, cognitive training games (CTGs). These programs usually comprise focused activities derived from specific scientific measures of cognitive function. This new kind of game has been extremely popular around the world, especially among older adults. Even if the primary intention is undoubtedly to launch a commercial product rather than to develop a scientifically validated intervention, these games may indeed have beneficial effects.

Previous studies showed that playing CTGs could improve cognitive functions such as memory and attention ( $\mathrm{Po}$ sitscience $^{7}$; Neuroracer ${ }^{8}$ ). However, these training programs were stand-alone software created in the laboratory for therapeutic purposes, with limited availability. For classical commercial brain training games, marketed to the larger public, evidence for beneficial effects on age-related deficits is less clear. ${ }^{9}$ Ackerman et al. ${ }^{10}$ investigated the transfer

${ }^{1}$ CIAMS, University of Paris Sud, University Paris-Saclay, Orsay Cedex, France.

${ }^{2}$ CIAMS, University of Orleans, Orléans, France.

${ }^{3}$ University Paris Descartes, Sorbonne Paris Cité, Laboratoire TEC, EA 3625, Paris, France.

${ }^{4}$ Scripps College, Claremont, California. 
effects of two types of activities (reading and CTG) that might mitigate age-related cognitive decline. A sample of 78 elderly completed twenty 1-hour training sessions with Big Brain Academy over the course of 1 month as well as twenty 1-hour reading sessions with articles on different current topics for another 1 month. Results showed no significant improvement from either the Big Brain Academy practice or the reading tasks in measures of cognitive and processing speed abilities.

Another study ${ }^{11}$ investigated the effects of twenty 1-hour CTG sessions with games selected from Lumosity on cognitive functions that decline with age. The results showed significant improvements in the trained group in processing speed and attention and no improvement in visuospatial working memory or executive control. These modest results on CTG could be explained by narrow and specific impact of cognitive training on trained abilities and nonexistent or very weak effect on abilities not targeted in the training. ${ }^{12,13}$ However, these previous studies are not precise enough in describing the content of the training games to verify this explanation.

Action videogame (AVG) is defined as videogames requesting speed, a high degree of perceptual, cognitive, and motor load in the service of an accurate motor plan, unpredictability, and an emphasis on peripheral processing. ${ }^{14}$ This kind of game offers highly variable environments and difficult tasks, as well as strong motivation and arousal. ${ }^{1}$ We know that AVG players show improvement in cognitive function, as well as changes in the brain substrates. ${ }^{15,16}$ An open question is whether there are differences in the beneficial impacts of CTGs and AVGs. Very few studies have compared the two.

In the domain of noncommercial CTG, Belchior et al. ${ }^{17}$ investigated the effect of videogame training on older adult's useful field of view (UFOV) performance. Older adults were randomized to receive practice with an AVG (Medal of Honor), a placebo control arcade game (Tetris), a clinically validated CTG UFOV training program, or into a no-contact control group. There was no difference between the three training conditions, but all were superior to the control group. In the domain of commercial brain training games, Nouchi et al. ${ }^{18}$ investigated the impact of a CTG, Brain Age, on cognitive functions in the elderly. Volunteers were randomly assigned to either of two game groups (Brain Age or Tetris). Participants in both groups played their game for about 15 minutes per day, at least 5 days per week, for 4 weeks. Results showed that playing the CTG improved executive functions and processing speed and not global cognitive status or attention.

The purpose of the present study was to compare a CTG (KBT) and an AVG (Super Mario Bros, SMB) in their effects on cognitive function in older adults. These two kinds of game present very different experiences of gameplay.

KBT is specifically designed to improve cognitive functions, especially in the elderly, with focused short games, each centered on one cognitive function (e.g., inhibition, working memory, and spatial orientation). SMB is a platform game, in which the gameplay revolves heavily around players controlling a character who runs and jumps onto platforms, floors, ledges, stairs, or other objects depicted on a single or scrolling (horizontal or vertical) game screen. Platform games are frequently classified as a subgenre of AVG. ${ }^{19,20}$ Players are simultaneously engaged in highly parallel processing involving several domains such as rapid shifts of visual attention, speeded decision making, and executive functions such as strategy selection and monitoring.

We hypothesized that both game programs would result in cognitive enhancement, but that the gameplay differences would lead to distinguishable benefits. KBT benefits would be greatest on tests that most closely mirrored the videogame. ${ }^{21}$ SMB would show benefits even to functions that were not specifically trained due to its highly variable environment.

\section{Materials and Methods}

\section{Participants}

Thirty-six independently living older adults (24 women, 12 men, from 60 to 71 years of age) volunteered to take part in this study. All participants gave written consent after having been informed as to the nature and purpose of the experiment which was approved by local ethics committees. The study conformed to the standards set by the Declaration of Helsinki. Inclusion criteria were to be between 60 and 72 years old, have no particular experience with videogames, report self-rated health status as fair, good, or excellent on a 5 -point health scale $(1=$ very bad, $2=$ bad, $3=$ fair, $4=$ good, and $5=$ excellent), have normal or corrected-to-normal vision and audition, and report themselves free of mental disorders, epilepsy, or color blindness. By random assignment, 12 participants were assigned to the KBT group, 12 participants to the SMB group, and 12 participants to a no-training, no contact control group.

Over the course of the study, one control participant could not attend the post-test session. The analyses were based on the 11 participants remaining in the control group. Demographic information is listed in Table 1 . There were no significant differences between the three groups in age, years of education, or self-rated health.

\section{Measures}

Three main categories of cognitive functions were tested: executive functions, processing speed, and visuospatial abilities. Executive functions are thought to be among the first cognitive functions to experience the negative effects of normal aging. ${ }^{2-24}$ These are also functions that have proved sensitive to improvement following the practice of videogames. ${ }^{6,25}$

Concerning processing speed, this function is a general resource underlying a good number of cognitive operations. Its slowing contributes significantly to the decline in cognitive performance with age. ${ }^{26}$ This category of functions has also

Table 1. Participant Demographic Characteristics

\begin{tabular}{|c|c|c|c|c|c|c|c|c|}
\hline \multirow[b]{2}{*}{ Variables } & \multicolumn{2}{|c|}{$\begin{array}{l}\text { Control } \\
(\mathrm{n}=11)\end{array}$} & \multicolumn{2}{|c|}{$\begin{array}{c}S M B \\
(\mathrm{n}=12)\end{array}$} & \multicolumn{2}{|c|}{$\begin{array}{c}K B T \\
(\mathrm{n}=12)\end{array}$} & \multirow[b]{2}{*}{$\mathrm{F}(2,32)$} & \multirow[b]{2}{*}{ ) $P$} \\
\hline & $M$ & $S D$ & $M$ & $S D$ & $M$ & $S D$ & & \\
\hline Age (years) & 65.55 & 2.91 & 63.75 & 2.49 & 64.67 & 3.17 & 1.52 & n.s \\
\hline $\begin{array}{l}\text { Self-rated } \\
\text { health }\end{array}$ & 3.55 & 0.52 & 3.92 & 0.67 & 3.75 & 0.45 & 1.28 & n.s \\
\hline $\begin{array}{l}\text { Years of } \\
\text { education }\end{array}$ & 11.36 & 1.57 & 12.00 & 2.92 & 11.91 & 2.84 & 0.21 & n.s \\
\hline
\end{tabular}

KBT, Kawashima Brain Training; M, mean; n.s., nonsignificant; SD, standard deviation; SMB, Super Mario Bros. 
been shown to be sensitive to videogames., ${ }^{3,5,6}$ Finally, visuospatial abilities decline as we age and are particularly sensitive to videogame stimulation. ${ }^{27,28}$

These three categories of functions are frequently chosen within studies of videogames ${ }^{11,18,29}$ because they represent critical functions when playing videogames (e.g., alternating attention between game stimuli, responding as quickly as possible, planning actions, and reasoning). All participants completed several cognitive tests: trail-making test ${ }^{30}$ (part B-part A) assessing flexibility, Stroop test ${ }^{31}$ (incongruent condition) assessing inhibitory mechanisms, matrix reasoning (subtest of $\mathrm{WASI}^{32}$ ) assessing reasoning, digit symbol substitution test (DSST) (subtest of WAIS- ${ }^{33}$ ) assessing processing speed, visuomotor coordination, and working memory, Corsi block test (subtest of $\mathrm{WMS}^{34}$ ) assessing spatial memory, number comparison test ${ }^{35}$ assessing processing speed, and spatial relations (subtest of DAT $5^{36}$ ) assessing visuospatial functions. Lezak et al. ${ }^{37}$ provide more complete descriptions of these tests.

\section{Procedure}

The entire study spanned 8-10 weeks. This included the pre- and post-test sessions for cognitive assessment and, for both training groups, the game sessions. At the first session, participants completed the battery of cognitive tests. Then, participants in the game groups were instructed to play the videogame (KBT or SMB) on a portable Nintendo Dual screen (DS) XXL console for 1 hour, thrice per week, over a period of 2 months, for a total training time of 24 hours. For both game groups, participants logged their sessions and scores in a follow-up book with schedule information. Instructions about how to use the Nintendo DS, the KBT, or SMB game and the follow-up log book were given to each game participant.

For the KBT group, participants were asked to play freely at games from two programs, KBT and Kawashima Brain Training Advanced. Participants were asked to regularly vary among the 20 available games. The training is typically broken down into subdomains, and games are highly similar in content and structure to tests used on psychological assessment scales (e.g., list learning to enhance semantic memory). To assess progress in KBT, two brain age assessments were recorded at the end of each 1-hour game session. The assessment option provides an evaluation of participants after they have trained in various tests. The tests changed randomly at each assessment session. Results in these tests are converted to a brain age by the software program.
For the SMB group, participants were asked to play freely at SMB and note which level they managed to reach during the 1hour game session. SMB requires simultaneous pursuit of several goals: finding the fastest possible path to cross a level, eliminating several types of enemies, and recovering a maximum of bonuses. When the twenty-four 1-hour game sessions were complete, participants were to contact the study coordinator to schedule the post-test session. Participants in the control group committed themselves not to modify their lifestyle and not to begin playing videogames over the 8 weeks of the study.

\section{Results}

To verify that both game and control groups were equivalent at the outset of the program, analysis of variances (ANOVAs) were used to compare the pretraining scores on each of the cognitive measures for the three groups (Table 2). Among the comparisons, none was significant.

Each participant of both game groups completed 24 sessions. In the KBT group, the follow-up logs showed that participants played regularly and with a variety of different games. Participants showed regular improvement in game performance across sessions. They passed from a mean cerebral age of 58.6 years at the beginning of training to 34.2 years at the end of training, $t(11)=6.72, P<0.0001$. In the SMB group, participants crossed on average eight levels (mean $=$ 8.08 , standard deviation $=5.05), t(11)=4.86, P<0.001$.

To determine whether both gaming regimens resulted in greater change than the untrained controls and whether KBT and SMB training led to different benefits, we calculated a change score by subtracting the pretest score from the posttest score for each cognitive measure. A multivariate ANOVA with all the measures within a set as dependent variables revealed a significant group effect, Wilk's $\Lambda=0.27$, $F(16,50)=2.94, P<0.01$. ANOVAs on each of the cognitive measures are shown in Table 3 . Tukey post hoc tests showed that the matrix reasoning change score was significantly greater in both game groups than in the control group. The Stroop test change was significantly greater in the KBT group than in control and SMB groups. The DSST, Corsi block test, spatial relations test, and number comparison test showed significantly greater change in the SMB group than in the control group with KBT intermediate.

\section{Discussion}

The purpose of the present study was to determine the potential of training based on two different types of sedentary videogame as a mode of activity that could have

Table 2. Pretraining Scores on Each of the Cognitive Measures for the Three Groups

\begin{tabular}{|c|c|c|c|c|c|c|c|c|}
\hline \multirow[b]{2}{*}{ Tests } & \multicolumn{2}{|c|}{ Control } & \multicolumn{2}{|c|}{$S M B$} & \multicolumn{2}{|c|}{$K B T$} & \multirow[b]{2}{*}{$\mathrm{F}(2,32)$} & \multirow[b]{2}{*}{$\mathrm{P}$} \\
\hline & $M$ & $S D$ & $M$ & $S D$ & $M$ & $S D$ & & \\
\hline Trail making test part B-part A (seconds) & 28.09 & 22.20 & 35.38 & 16.81 & 31.30 & 17.24 & 0.43 & \\
\hline Stroop: incongruent (no.) & 42.55 & 10.61 & 47.00 & 7.20 & 41.17 & 13.00 & 0.99 & \\
\hline Matrix reasoning test (no.) & 21.30 & 5.35 & 22.42 & 4.66 & 21.50 & 7.65 & 0.12 & \\
\hline Digit-symbol substitution (no.) & 69.73 & 11.17 & 73.08 & 10.4 & 72.58 & 11.67 & 0.30 & \\
\hline Corsi block test (no.) & 4.73 & 0.91 & 4.58 & 1.24 & 4.58 & 1.24 & 0.06 & \\
\hline Spatial relations (no.) & 14.00 & 6.00 & 11.83 & 5.29 & 11.75 & 6.55 & 0.52 & n.s \\
\hline No. comparison (no.) & 29.27 & 6.12 & 27.67 & 6.92 & 28.5 & 4.19 & 0.22 & \\
\hline
\end{tabular}


Table 3. Change Scores, Analysis of Variance, and Tukey's Post Hoc Tests on Each Cognitive Measure for the Three Groups

\begin{tabular}{|c|c|c|c|c|c|c|c|c|c|c|c|c|}
\hline \multirow[b]{2}{*}{ Tests } & \multicolumn{2}{|c|}{ Control } & \multicolumn{2}{|c|}{$S M B$} & \multicolumn{2}{|c|}{$K B T$} & \multirow{2}{*}{$\underset{(2,32)}{\mathrm{F}}$} & \multirow[b]{2}{*}{$\mathrm{P}$} & \multicolumn{4}{|c|}{ Tukey's post hoc } \\
\hline & $M$ & $S D$ & $M$ & $S D$ & $M$ & $S D$ & & & $K B T>C$ & $S M B>C$ & $S M B>K B T$ & $K B T>S M B$ \\
\hline $\begin{array}{l}\text { Trail making test part } \\
\text { B-part A (seconds) }\end{array}$ & 0.36 & 4.41 & -1.15 & 15.09 & 2.86 & 17.20 & 0.26 & n.s & n.s & n.s & n.s & n.s \\
\hline Stroop: incongruent (no.) & 0.91 & 3.81 & 2.75 & 4.85 & 9.25 & 6.30 & 8.56 & $<0.01$ & $<0.01$ & n.s & n.s & $<0.05$ \\
\hline Matrix reasoning test (no.) & -1.91 & 1.58 & 3.42 & 4.03 & 2.08 & 4.50 & 6.54 & $<0.01$ & $<0.05$ & $<0.05$ & n.s & n.s \\
\hline $\begin{array}{l}\text { Digit-symbol } \\
\text { substitution (no.) }\end{array}$ & -1.55 & 2.77 & 7.25 & 8.19 & 3.75 & 7.45 & 5.03 & $<0.05$ & n.s & $<0.01$ & n.s & n.s \\
\hline Corsi block test (no.) & -0.09 & 0.83 & 0.75 & 0.75 & 0.67 & 0.89 & 3.56 & $<0.05$ & n.s & $=0.05$ & n.s & n.s \\
\hline Spatial relations (no.) & -1.09 & 2.21 & 1.83 & 2.55 & -0.83 & 3.49 & 3.89 & $<0.05$ & n.s & $<0.05$ & $=0.06$ & n.s \\
\hline No. comparison (no.) & -1.73 & 2.49 & 2.25 & 5.24 & 0.42 & 2.71 & 3.26 & $<0.05$ & n.s & $<0.05$ & n.s & n.s \\
\hline
\end{tabular}

KBT $>C$, Kawashima Brain Training progress is superior to group control progress; KBT $>$ SMB, Kawashima Brain Training progress is superior to Super Mario Bros progress; SMB $>$ C, Super Mario Bros progress is superior to group control progress; SMB $>$ KBT, Super Mario Bros progress is superior to Kawashima Brain Training progress.

cognitive benefits for older adults. Training with both the AVG (SMB) and the CTG (KBT) led to significant improvements in some aspects of cognitive functions. Both $\mathrm{SMB}$ and KBT promoted inductive reasoning (matrix reasoning test), despite their quite different natures. This conclusion is similar to previous studies, which found benefits of videogame training on executive functions, both with CTG programs ${ }^{18,38}$ and with entertainment videogames (for a review Ref. ${ }^{29}$ ).

The scope of benefits of SMB training seems broader than those from the KBT program: the CTG resulted in less transfer to cognitive tasks than did the AVG. This result is consistent with previous results ${ }^{10}$ which found limited evidence for effects of CTG training on cognition. The intrinsic characteristics of SMB, such as task variability, task difficulty, and elicited motivation and arousal may well be partly responsible for cognitive improvements. ${ }^{1}$ The SMB environment is rich and highly variable, involving alternation among a wide range of tasks.

As pointed out by Green and Bavelier, ${ }^{1}$ entertainment games can be exceedingly complex and do tap many systems in parallel. SMB gamers are simultaneously challenged in speed, accuracy, reasoning, and strategy. An important aspect of what gamers may learn from SMB and similar AVG is the ability to distribute their skills among multiple tasks with different processing priorities and consequently develop the accommodation of their skills to different tasks. Green and Bavelier ${ }^{1}$ suggest that the more a program calls on a variety of combinations of processes, the greater the observed transfer to cognitive function.

By contrast, KBT is a single-task environment. The training is typically broken down into subdomains with, for example, inhibition trained separately from speed of processing. This kind of training leads to efficient near transfer to a closely-related assessment task (as in inhibition) but lesser far transfer to different tasks. ${ }^{39}$ Moreover, the CTG game situations are not characterized by high variability and alternation between tasks. The limited variability may induce learning that is specific to the tasks being learned and that does not generalize well to new tasks or variants of the same task. ${ }^{40}$ In addition, the natural attractiveness of SMB is another factor that could have aided in cognitive transfer. ${ }^{41,42}$
The scientific aura of the KBT program could reduce attractiveness of the game (e.g., mentions of terms such as professor, brain age, and magnetic resonance imaging in the introduction). KBT participants may have the impression that they are training, whereas SMB participants feel they are simply playing.

This study presents several limits. First, the SMB program seems to be more effective than the KBT program, significantly improving more cognitive functions. However, there was only one significant difference in improvement between the two programs. Then, one of the objectives of this study was to observe the impact of videogames when played freely. This ecologically valid approach has an essential limit. Indeed, the training was not perfectly standardized among participants; each could have focused on different aspects of the game.

Although cognitive function was homogeneous across the three groups before the intervention and although the observed scores were within the normal range for those tasks with norms available, the lack of a global measure of cognitive function such as the Mini Mental State Examination does allow the possibility that the sample was not normative and that the generalizability of the results may be circumscribed.

Last, each game category is represented by only one game. It would be interesting to replicate this study with other games from the same categories (e.g., fighting games for AVG and independent computer software like positscience for CTG). Thus, we would be able to establish if the present results are specific to the two selected games (KBT and SMB) and to the stimulated cognitive functions or if they can be generalized to the game categories (AVG and CBT).

It would have been interesting to measure the expectations for each group to assess how these expectations influenced the training effects. Another direction for future research is to examine the consequences of training for everyday competencies such as meal preparation and shopping. ${ }^{\text {. This kind }}$ of transfer is rarely tested in training research but, as it directly addresses quality of life, it should be investigated more extensively.

One particular class of games has shown substantial benefits to cognitive function in older adults, exergames. ${ }^{29} \mathrm{Ex}-$ ergames are innovative and interactive digital games that 
combine physical exercise and videogames. It would be interesting to include exergames, AVG, and CTG in the same design, to determine the differences between sedentary and active videogames on cognitive functioning in older adults and to assess the added value of the physical stimulation only present in exergame group. ${ }^{29,43}$

\section{Acknowledgments}

The authors thank the participants in this study and Amandine Amistani and Marc Delfosse, Master's students, for their contributions.

\section{Author Disclosure Statement}

No competing financial interests exist.

\section{References}

1. Green CS, Bavelier D. Exercising your brain: A review of human brain plasticity and training-induced earning. Psychol Aging 2008; 23:692-701.

2. Spence I, Feng J. Video games and spatial cognition. Rev Gen Psychol 2010; 14:92-104.

3. Goldstein J, Cajko L, Oosterbroek M, et al. Video games and the elderly. Soc Behav Personal 1997; 25:345-352.

4. Drew D, Waters J. Video games: Utilization of a novel strategy to improve perceptual motor skills and cognitive functioning in the non-institutionalized elderly. Cognitive Rehab 1986; 4:26-31.

5. Dustman RE, Emmerson RY, Steinhaus LA, et al. The effects of videogame playing on neuropsychological performance of elderly individuals. J Gerontol 1992; 47:168171.

6. Basak C, Boot WR, Voss MW, Kramer AF. Can training in a real-time strategy videogame attenuate cognitive decline in adults? Psychol Aging 2008; 23:765-777.

7. Smith GE, Housen P, Yaffe K, et al. A cognitive training program based on principles of brain plasticity: Results from the improvement in memory with plasticity-based adaptive cognitive training (IMPACT) study. J Am Geriatr Soc 2009; 57:594-603.

8. Anguera JA, Boccanfuso J, Rintoul JL, et al. Video game training enhances cognitive control in older adults. Nature 2013; 501:97-101.

9. Simons DJ, Boot WR, Charness N, et al. Do 'braintraining' programs work? Psychol Sci Public Interest 2016; 17:103-186.

10. Ackerman PL, Kanfer R, Calderwood C. Use it or lose it? Wii brain exercise practice and reading for domain knowledge. Psychol Aging 2010; 25:753-766.

11. Ballesteros S, Prieto A, Mayas J, et al. Brain training with non-action video games enhances aspects of cognition in older adults: A randomized controlled trial. Front Aging Neurosci 2014; 6:277.

12. Melby-Lervag M, Hulme C. Is working memory training effective? A meta-analytic review. Dev Psychol 2013; 49: 270-291.

13. Sims VK, Mayer, RE. Domain specificity of spatial expertise: The case of video game players. Appl Cognit Psychol 2002; 16:97-115.

14. Green CS, Li R, Bavelier D. Perceptual learning during action video game playing. Top Cogn Sci 2010; 2:202216.
15. Nagamitsu S, Nagano M, Yamashita Y, et al. Prefrontal cerebral blood volume patterns while playing video games-A near-infrared spectroscopy study. Brain Dev 2006; 28:315-321.

16. Kuhn S, Gleich T, Lorenz RC, et al. Playing super mario induces structural brain plasticity: Gray matter changes resulting from training with a commercial video game. Mol Psychiatry 2014; 19:265-271.

17. Belchior P, Marsiske M, Sisco S, et al. The impact of video game training on selected visual attention in older adults. Comput Human Behav 2013; 29:1318-1324.

18. Nouchi R, Taki Y, Takeuchi H, et al. Brain training game improves executive functions and processing speed in the elderly: A randomized controlled trial. PLoS One 2012; 7: e29676.

19. Arsenault D. Video game genre, evolution and innovation. Eludamos J Comput Game Culture 2009; 3:149-176.

20. Typology of video games, Hermes, the review 2012; 1:15-16.

21. Mahncke HW, Connor BB, Appelman J, et al. Memory enhancement in healthy older adults using a brain plasticitybased training program: A randomized, controlled study. Proc Natl Acad Sci U S A 2006; 103:12523-12528.

22. Stuss D, Alexander M. Executive functions and the frontal lobes: A conceptual view. Psychol Res 2000; 63: 289-298.

23. Raz N. Aging of the brain and its impact on cognitive performance: Integration of structural and functional findings. In: Craik FIM, Salthouse TA, eds. Handbook of Aging and Cognition-II. Mahwah, NJ: Erlbaum; 2000, pp.1-90.

24. West R. An application of prefrontal cortex function theory to cognitive aging. Psychol Bul 1996; 120:272-292.

25. Boot WR, Kramer AF, Simons DJ, et al. The effects of video game playing on attention, memory, and executive control. Acta Psychol 2008; 129:387-398.

26. Salthouse TA. The processing-speed theory of adult age differences in cognition. Psychol Rev 1996; 103:403-428.

27. Green CS, Bavelier D. Action video games modify visual selective attention. Nature 2003; 423:534-537.

28. Green CS, Bavelier D. Effects of action video game playing on the spatial distribution of visual selective attention. J Exp Psychol Hum Percept Perform 2006; 32 : 1465-1478.

29. Maillot P, Perrot A, Hartley A. Effects of interactive physical-activity video-game training on physical and cognitive function in older adults. Psychol Aging 2012; 27: 589-600.

30. Corrigan JD, Hinkeldey NS. Relationships between parts A and B of the Trail Making Test. J Clin Psychol 1987; 43: 402-409.

31. Stroop JR. Studies of interference in serial verbal reactions. J Exp Psychol 1935; 18:643-662.

32. Wechsler D. Wechsler Abbreviated Scale of Intelligence (WASI). San Antonio, TX: Harcourt Assessment; 1999.

33. Wechsler D. Wechsler Adult Intelligence Scale-3rd Edition (WAIS-3R). San Antonio, TX: Harcourt Assessment; 1997.

34. Wechsler D. Wechsler Memory Scale-3rd Edition (WMSIII). San Antonio, TX: Psychological Corporation; 1997.

35. Ackerman PL, Cianciolo AT. Cognitive, perceptual speed, and psychomotor determinants of individual differences during skill acquisition. J Exp Psychol Applied 2000; 6: 259-290.

36. Bennet GK, Seashore G, Wesman AG. DAT: Differential Aptitude Test. New York: The Psychological Corporation. 
Adaptation française, Tests différentiels d'aptitudes. Paris: ECPA; 1974.

37. Lezak MD, Howieson DB, Loring DW, et al. Neuropsychological Assessment, 4th ed. New York, NY: Oxford University Press; 2004.

38. van Mujden J, Band GPH, Hommel B. Online games training aging brains: Limited transfer to cognitive control functions. Front Hum Neurosci 2012; 6:1-13.

39. Ahissar M, Hochstein S. The reverse hierarchy theory of visual perceptual learning. Trends Cogn Sci 2004; 8:457464.

40. Gómez RL. Variability and detection of invariant structure. Psychol Sci 2002; 13:431-436.

41. Graves LE, Ridgers ND, Williams K, et al. The physiological cost and enjoyment of Wii Fit in adolescents, young adults, and older adults. J Phy Act Health 2010; 7: 393-401.
42. Herzog MH, Fahle M. The role of feedback in learning a vernier discrimination task. Vision Res 1997; 37:21332141.

43. Anderson-Hanley C, Arclero PJ, Brickman AM, et al. Exergaming and older adult cognition. A cluster randomized clinical trial. Am J Prev Med 2012; 42:109-119.

Address correspondence to: Alexandra Perrot, PhD UFR STAPS University of Paris Sud University Paris-Saclay, Orsay Cedex, France

E-mail: alexandra.perrot@u-psud.fr 